\title{
Properties of Immobile Hydrogen Confined in Microporous Carbon
}

\author{
Jitendra Bahadur ${ }^{1,2}$, Cristian I. Contescu ${ }^{3 *}$, Anibal J. Ramirez-Cuesta ${ }^{4 *}$, Eugene Mamontov ${ }^{4 *}$, \\ Nidia C. Gallego ${ }^{3}$, Yongqiang Cheng ${ }^{4}$, Luke L. Daemen ${ }^{4}$, Yuri B. Melnichenko ${ }^{2}$ \\ ${ }^{1}$ Biology and Soft Matter Division, Oak Ridge National Laboratory, Oak Ridge, TN 37831, \\ United States \\ ${ }^{2}$ Solid State Physics Division, Bhabha Atomic Research Centre, Mumbai, 400085, India. \\ ${ }^{3}$ Materials Science and Technology Division, Oak Ridge National Laboratory, Oak Ridge, \\ TN 37831, United States \\ ${ }^{4}$ Chemical and Engineering Materials Division, Oak Ridge National Laboratory, Oak Ridge, \\ TN 37831, United States
}

\begin{abstract}
We report results of vibrational neutron spectroscopy investigation aimed to identify the state of hydrogen adsorbed in ultramicroporous carbon. The mobility of hydrogen confined in carbon pores was probed as a function of temperature and pressure using inelastic neutron scattering, and the molecular translational and rotational motions were studied. At low loading rotation of $\mathrm{H}_{2}$ molecules adsorbed in the smallest carbon pores ( 4-5 $\AA$ ) is severely hindered, suggesting that the interaction between $\mathrm{H}_{2}$ and the host matrix is anisotropic. At higher loading, $\mathrm{H}_{2}$ molecules behave as a nearly free rotor, implying lower anisotropic interactions with adsorption sites. At $77 \mathrm{~K}$ where bulk $\mathrm{H}_{2}$ is a gas, deconvolution of elastic/quasielastic signal provide evidence of pressure-dependent fractions of immobile (solid-like) and partially mobile (liquid-like) hydrogen, which correlate with the excess adsorption isotherm at $77 \mathrm{~K}$. Effective $\mathrm{H}_{2}$ density in pores changes from solid-like to liquidlike with increasing pressure at $77 \mathrm{~K}$. Surprisingly, immobile and partially mobile $\mathrm{H}_{2}$ is present even at temperatures as high as $\sim 110 \mathrm{~K}$ where bulk hydrogen exists only in gas form.
\end{abstract}

\footnotetext{
*Corresponding authors. Emails: ContescuCI@ ornl.gov (Cristian I. Contescu), RamirezCueAJ@ornl.gov (Anibal J. Ramirez-Cuesta)

This manuscript has been authored by UT-Battelle, LLC under Contract No. DE-AC05-000R22725 with the U.S. Department of Energy. The United States Government retains and the publisher, by accepting the article for publication, acknowledges that the United States Government retains a non-exclusive, paid-up, irrevocable, world-wide license to publish or reproduce the published form of this manuscript, or allow others to do so, for United States Government purposes. The Department of Energy will provide public access to these results of federally sponsored research in accordance with the DOE Public Access Plan (http://energy.gov/downloads/doe-public-access-plan).
} 


\section{Introduction}

Hydrogen storage remains the primary bottleneck to be overcome for the successful implementation of hydrogen-based technology in various applications, and is still a major challenge for materials science. In order to find better hydrogen storage materials, different porous materials have been discovered and studied over the years [1-5]. The results showed that the uptake of physisorbed hydrogen is closely connected to the available pore volume and surface area of porous materials. It was found that the presence of micropores $(<2 \mathrm{~nm})$, and in particular ultramicropores $(<0.7 \mathrm{~nm})$, is most desirable for hydrogen adsorption [6-14], in perfect agreement with theoretical modeling results $[15,16]$. Carbon materials are among the most investigated adsorbent materials [17] because of their low density, extensive pore structure, chemical stability, low cost, accessibility, and recyclability [18]. Electron microscopy results show that activated carbons consist of isotropic three dimensional networks of wrinkled atomic thin graphene sheets, and that the irregular stacking of crumpled sheets forms micropores of few $\AA$ in size [19,20]. The large variation in pore morphology results from differences in raw materials, carbonization, and activation methods $[21,22]$. Identifying strong adsorption sites and the adsorption mechanism in the microporous activated carbon is necessary to design better host materials for hydrogen storage.

This work presents a neutron vibrational spectroscopy investigation of molecular hydrogen confined in microporous carbon. Vibrational spectroscopy [23] is a well-known analytical technique that provides information on structure, bonding, and interactions in chemical systems, with the most popular examples being infrared (FTIR) and Raman spectroscopy techniques. Using neutrons in vibrational spectroscopy studies on hydrogen adsorption takes advantage of the particularly high sensitivity of neutron scattering by hydrogen species. The high penetration of neutrons in solid materials allows studies on adsorbed species and in-pore 
confined molecular species as well. Moreover, neutron scattering instruments use a variety of sample environments, including in-situ gas adsorption from high vacuum to high pressures, and controlled temperatures from cryogenic to elevated temperatures. Depending on the type of neutron spectrometers it is possible to investigate a multitude of properties in the hydrogen / carbon system. By small-angle neutron scattering (SANS) [24,25] it has been found that the density of $\mathrm{H}_{2}$ in micropores is about twenty times higher than the bulk gas at $~ 35$ bar and room temperature [25]. Higher pressure ( 200 bar) results obtained by SANS at room temperature showed that $\mathrm{H}_{2}$ adsorbed in narrow micropores $(<9 \AA)$ reaches the density of liquid hydrogen [24]. These experimental results are in line with empirical estimates according to which $\mathrm{H}_{2}$ adsorbed in narrow micropores is in a highly dense state [26], most likely liquid [27]. Theoretical predictions for $\mathrm{H}_{2}$ in narrow slit-shaped carbons led to the same result $[28,29]$. Challenging the view that the highest densification state of supercritical hydrogen in carbon micropores is the liquid state $[9,27]$, a recent inelastic neutron scattering (INS) study provided direct evidence of solid-like hydrogen immobilized at $77 \mathrm{~K}$ at pressures as low as 0.2 bar [30]. Prompted by this result, the main purpose of this work is to further investigate the physical state of $\mathrm{H}_{2}$ adsorbed in carbon pores using dynamic neutron scattering methods over a broader range of pressures and temperatures. The dynamics of adsorbed $\mathrm{H}_{2}$ molecules as a function of thermodynamic parameters such as temperature and pressure reveals the nature of the interaction between $\mathrm{H}_{2}$ and the host matrix, and provides insight into the actual physical state of adsorbed $\mathrm{H}_{2}$. Recently, quasielastic neutron scattering (QENS) studies on the dynamics of $\mathrm{H}_{2}$ and $\mathrm{D}_{2}$ confined in carbon micropores showed that the diffusion onset temperature is higher for $\mathrm{H}_{2}$ than for $\mathrm{D}_{2}$ [31]. This observation, which is the opposite to what is predicted by classical physics, is the consequence of quantum behavior at cryogenic temperatures of $\mathrm{H}_{2}$ and $\mathrm{D}_{2}$ molecules restricted in the overlapping potential of pore walls in ultramicropores. The QENS study suggests that classical notions of liquid and solid 
have different signification when applied to $\mathrm{H}_{2}$ adsorbed in sub-nanometer sized pores [32]. It is important to note that the information on diffusive motion of adsorbed molecules provided by QENS technique is obtained from high resolution measurements $(\sim \mu \mathrm{eV})$ of the scattering signal broadening near the elastic peak. It conveys information on both mobile and immobile species. However at higher temperatures it is difficult to determine both components if the instrument is a high resolution QENS spectrometer, since most of the diffusive behaviors will be outside the instrument measurement window. In an inelastic neutron scattering (INS) spectrometer it could be possible to determine the total contribution to the intensity due to both species, although the resolution is oftentimes not good enough to deconvolute the elastic and quasielastic contributions.

The main finding of this paper is the persistence of immobile (solid-like) and partially mobile (liquid-like) $\mathrm{H}_{2}$ confined in carbon pores over a broad range of pressures and temperatures, including high pressure (up to 40 bar) and supercritical temperatures (up to $110 \mathrm{~K}$ ). The nature of the interaction potential between $\mathrm{H}_{2}$ and adsorption sites has been probed by monitoring the para $\rightarrow$ ortho transition as a function of temperature, pressure, and $\mathrm{H}_{2}$ loading. In addition, a quasielastic analysis at low energy transfers was performed to the extent afforded by the spectrometer limitations. From the quasielastic signal broadening around the elastic line it was possible to estimate the proportion of immobile (solid-like) and partially mobile (liquid-like) species as a function of surface loading at $77 \mathrm{~K}$. The results showed that the effective density of pore-confined $\mathrm{H}_{2}$ at $77 \mathrm{~K}$ depends strongly on pressure and local pore width. This work demonstrates that the previous observation by one of the co-authors of immobile $\mathrm{H}_{2}$ above its critical point [30] was not an isolated finding, but a part of a general behavior of in-pores confined $\mathrm{H}_{2}$. The significance of these findings reaches beyond the immediate interest in adsorption theory and confined fluids behavior, and is expected to infuse a new impetus into the search for the optimal hydrogen storage material. 


\section{Experimental}

Ultramicroporous carbon (UMC) is a chemically activated porous carbon made available by MeadWestvaco Corporation (Charleston, SC) [33]. Gas adsorption characterization of surface properties [22] established that UMC has high surface area $\left(S_{B E T}=2450 \mathrm{~m}^{2} / \mathrm{g}\right)$ and high total pore volume $\left(V_{t o t}=1.19 \mathrm{~cm}^{3} / \mathrm{g}\right)$. The pore size distribution calculated from $\mathrm{N}_{2}$ and $\mathrm{CO}_{2}$ adsorption is presented in Figure S1 of Supporting Information. About $70 \%$ of $V_{\text {tot }}$ is contained in micropores $(<20 \AA)$, and $20 \%$ of $V_{\text {tot }}$ comes from ultramicropores $(<7 \AA)$. Quantitative analysis of SANS patterns recorded for the "dry" UMC material (in vacuum, before exposure to hydrogen) produced similar conclusions [34]. Hydrogen adsorption on UMC shows unusually high uptake capacity at $25{ }^{\circ} \mathrm{C}(0.8 \mathrm{wt} \%$ at 20 bar) with slow kinetics and desorption hysteresis [22,25]. Adsorption of $\mathrm{H}_{2}$ at $77 \mathrm{~K}$ reaches 5.5 wt $\%$ at 20 bar, which is comparable with the best known activated carbons (e.g. Maxsorb, AX-21) often referenced as standards for cryogenic $\mathrm{H}_{2}$ physisorption. The excess adsorption data measured volumetrically (up to 1 bar) and gravimetrically (up to 20 bar) at $77 \mathrm{~K}$ are shown in Figure S2 of Supporting Information. High pressure adsorption data were fitted using with the Unilan isotherm, which is an extension of the Langmuir model for patch-like surfaces consisting of energetically uniform patches [35]. Using this model is was possible to extrapolate the adsorption isotherm at $77 \mathrm{~K}$ up to the highest pressure (40 bar) of current INS measurements. The Unilan parameters were used to estimate the $\mathrm{H}_{2}$ loading, $q_{P}$, after exposure to various pressures $(P)$ at $77 \mathrm{~K}$. The corresponding relative surface coverage degree, $\theta_{P}$, was calculated by normalizing to the asymptotic saturated adsorption amount $\left(q_{s}=32.5 \mathrm{mmol} / \mathrm{g}\right)$ obtained from fitting the Unilan isotherm. Details are available in the Supporting Information file. 
Inelastic neutron spectroscopy (INS) measurements were carried out on the VISION spectrometer at the Spallation Neutron Source at Oak Ridge National Laboratory. VISION is a particularly powerful neutron vibrational spectroscopy instrument, very similar to its optical counterparts - FTIR and Raman spectrometers - but with a remarkable sensitivity to hydrogen species in a very flexible sample environments. With a broad energy range extending from -3 up to $1000 \mathrm{meV}$ (or $8065 \mathrm{~cm}^{-1}$ ) VISION offers good resolution $(0.10 \mathrm{meV}$ around the elastic line and $1.2 \% \Delta E / E$ above $5 \mathrm{meV}$ ) in a range of momentum transfer vector $Q\left(1.3\right.$ to $\left.30 \AA^{-1}\right)$ that corresponds to physical lengths between 0.2 and $4.2 \AA$ [36]. Thus, this instrument is optimized for high $Q$ vibrational studies over broad energy ranges. The closest instrument of the same type is the TOSCA apparatus in UK. VISION measures the time of flight of each neutron for a fixed geometry configuration and integrates the signal within certain values of $Q$. Thus the $Q$ vector information is not available in VISION, and generally it is not required for vibrational spectroscopy. Moreover, the distribution of all resolution components is not strictly Gaussian [36].

The UMC sample in powder form $(0.5 \mathrm{~g})$ was outgassed for 20 hours at $300{ }^{\circ} \mathrm{C}$ (residual pressure $<0.1 \mathrm{~Pa})$, sealed into a cylindrical $\mathrm{Al}$ can $(6 \mathrm{~mm}$ diameter $)$ and subsequently loaded into a closed cycle He refrigerator. The INS spectrum of "dry" UMC was acquired in the range of 5 to $77 \mathrm{~K}$ and was used for background subtraction. Figure 1 shows a schematic of experimental conditions employed in this work and their relationship with the phase diagram of bulk $\mathrm{H}_{2}$. 


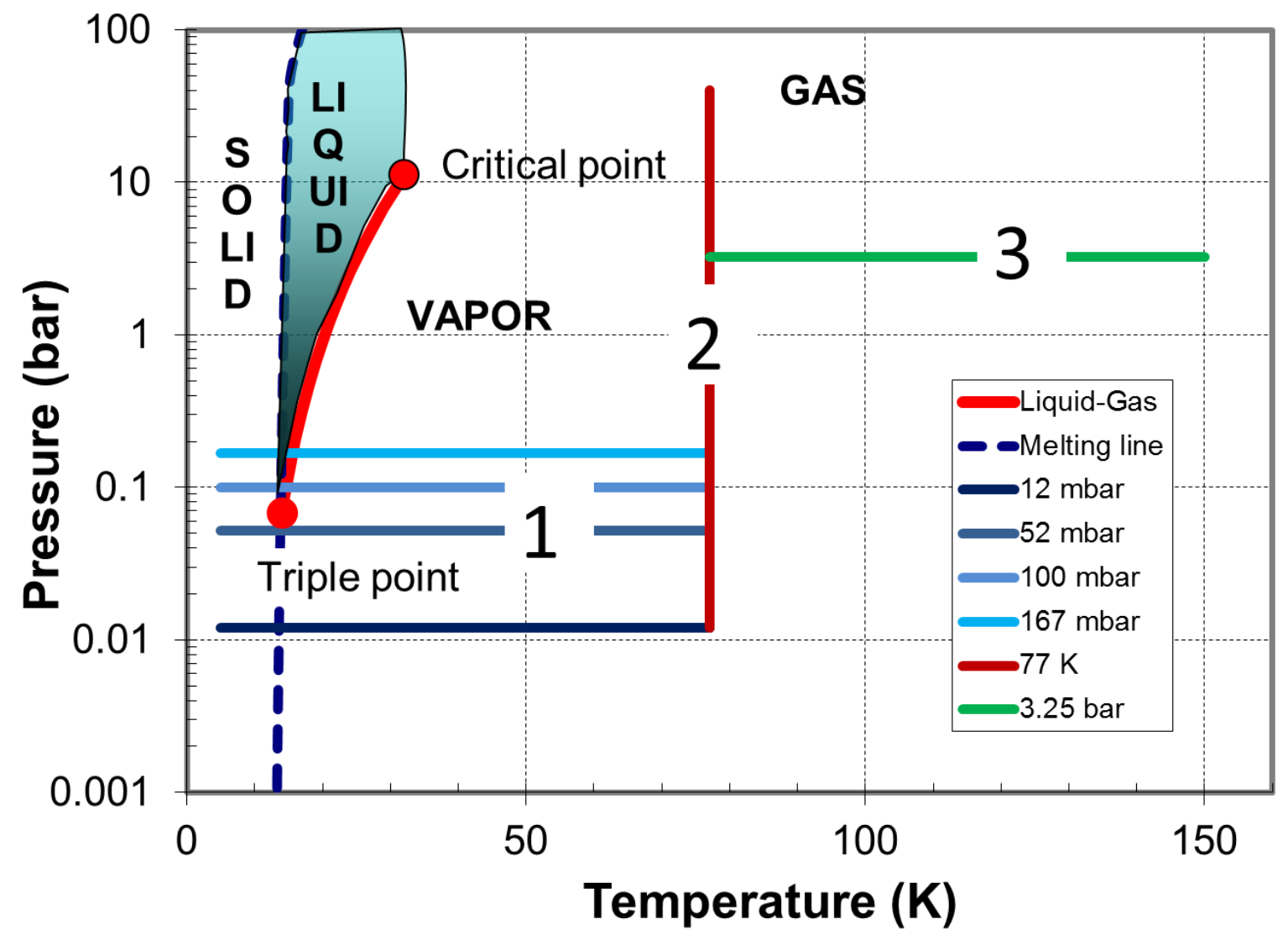

Figure 1: Experimental conditions in the three series of experiments (indicated by numbers) and their relationship with the phase diagram of bulk hydrogen [37].

Three series of experiments were performed. The first series comprised four runs at various sub-atmospheric pressures for studying the effect of temperature on hydrogen adsorbed at low surface coverage. These experiments used $\mathrm{p}-\mathrm{H}_{2}$ obtained by conversion of $99.9995 \%$ purity normal hydrogen $\left(\mathrm{n}-\mathrm{H}_{2}\right)$ over $\mathrm{Cr}_{2} \mathrm{O}_{3}$ catalyst at $20 \mathrm{~K}$. The present set-up can provide up to $99.5 \%$ of $\mathrm{p}-\mathrm{H}_{2}$. The $\mathrm{p}-\mathrm{H}_{2}$ was injected over the UMC sample at $77 \mathrm{~K}$ at fixed pressures $(12,52,100$ and 167 mbar) and allowed to equilibrate for one hour. Equilibration was accompanied by partial conversion to $\mathrm{o}-\mathrm{H}_{2}$ mediated by the paramagnetic centers present in UMC. The sample cell was then isolated, cooled down to $5 \mathrm{~K}$, and the INS spectra were recorded temperatures in the range of 5-77 K. In the second series of experiments the effect 
of $n-H_{2}$ pressure at $77 \mathrm{~K}$ was studied up to 40 bar. High purity $n-H_{2}$ was introduced to the sample at $77 \mathrm{~K}$ at various pressures and the sample pressure was kept constant during data acquisition by keeping the sample cell in contact with the gas reservoir. In a third series, the temperature effect on adsorbed $\mathrm{n}-\mathrm{H}_{2}$ was studied at a constant pressure of 3.25 bar and temperatures from $77 \mathrm{~K}$ to $150 \mathrm{~K}$. The inelastic spectra of adsorbed $\mathrm{H}_{2}$ were obtained by subtracting the spectrum of "dry" UMC from the spectra of hydrogen-loaded UMC.

\section{Theoretical Background}

Solid $\mathrm{H}_{2}$ behaves as a three-dimensional quantum rotor with energy levels given by

$$
E_{J}=B J(J+1)
$$

Here, $J$ is the quantum rotational number $(J=0,1, \ldots)$, and $B$ is the rotational constant of hydrogen defined as

$$
B=\frac{\hbar^{2}}{2 \mu d_{e}^{2}}=7.35 \mathrm{meV}
$$

where $\mu$ is the reduced mass of the molecule, and $d_{e}$ is the equilibrium distance between the two protons ( $0.741 \AA$ in the free state) [38]. The two nuclear-spin isomers of molecular $\mathrm{H}_{2}$, namely ortho-hydrogen and para-hydrogen, differ by the parity of rotational quantum number, as required by the total wave function symmetry with respect to the permutation of the two nuclei. The states with even $J$ values are called para-hydrogen $\left(\mathrm{p}-\mathrm{H}_{2}\right)$ while those with odd $J$ values are called ortho-hydrogen $\left(\mathrm{o}-\mathrm{H}_{2}\right)$. The rotational states are spin-locked, meaning that isolated $\mathrm{p}-\mathrm{H}_{2}$ molecule cannot convert into $\mathrm{o}-\mathrm{H}_{2}$. The conversion is slow in gas phase, by readily happens in the presence of paramagnetic sites on surfaces, and in such case the relative population of both species is determined by temperature [39]. 
Neutrons have spin and their interaction with $\mathrm{H}_{2}$ molecules (i.e., in the INS) can cause para $(J=0) \rightarrow$ ortho $(J=1)$ transition. This transition is observed [40] in solid $\mathrm{H}_{2}$ and for $\mathrm{H}_{2}$ adsorbed on the surface of different materials, e.g. Grafoil [41]. In all these cases the transition takes place at an energy $2 B=14.7 \mathrm{meV}$ as expected for a $3-\mathrm{D}$ free quantum rotor. Since the rotational energy of molecular $\mathrm{H}_{2}$ is comparable with the adsorption energy of physisorbed hydrogen (about $30 \mathrm{meV}$ per $\mathrm{H}_{2}$ molecule) [42-44] the rotational motion is retained after physisorption, but its character is altered by the adsorption potential. In a weak isotropic potential, adsorbed $\mathrm{H}_{2}$ continues to behave as a free rotor. If the potential is anisotropic the rotational symmetry is perturbed. If the adsorption potential anisotropy is small, the effect is a small shift of the rotational level and $\mathrm{H}_{2}$ behaves as a nearly free rotor. In the presence of a large anisotropic potential, the degeneracy of the $J=1$ state of $\mathrm{H}_{2}$ molecule is lifted and the rotational line splits. This hindered rotational motion is frequently observed for $\mathrm{H}_{2}$ adsorbed in microporous materials with pore sizes close to the molecular diameter [45,46]. As the anisotropic potential becomes infinitely large, the rotational motion tends to be confined in either the surface-normal or parallel direction, depending on the sign of anisotropic potential, leading to one-dimensional or two-dimensional rotation, respectively [47,48]. Thus, the $J=0 \rightarrow 1$ (para $\rightarrow$ ortho) rotational transition can be used as a probe of the interaction potential of $\mathrm{H}_{2}$ with adsorption sites. INS is quite sensitive to the dynamics of molecular $\mathrm{H}_{2}$ due to its large spin incoherent neutron cross-section, and in particular to the rotational transitions [49]. The INS technique has been widely used to probe the molecular hydrogen confined in the carbon-based porous materials including single-walled carbon nanotubes and nanohorns [45,46,50-57]. It has been shown in these studies that the presence of different adsorption sites for $\mathrm{H}_{2}$ causes energy shifts of the rotational peak. 


\section{Results and Discussion}

Figure 2 shows a typical inelastic spectrum of adsorbed $\mathrm{H}_{2}$ at $25 \mathrm{~K}$ and 167 mbar loading. The energy transfer range is from -2 to $1000 \mathrm{meV}$. The cross-section for elastic neutron scattering from $\mathrm{p}-\mathrm{H}_{2}$ is negligible compared to that of o- $\mathrm{H}_{2}$ (see Figure $\mathrm{S} 3$ of Supplementary Information) $[58,59]$. The occurrence of the elastic peak confirms that some fraction of $\mathrm{p}-\mathrm{H}_{2}$ delivered by the catalytic convertor was converted back into $\mathrm{o}-\mathrm{H}_{2}$ by the paramagnetic impurities in UMC. The zoomed spectrum in the inset shows the rotational peak at $14.6 \mathrm{meV}$ corresponding to para $\rightarrow$ ortho transition. Broad recoil peaks are observed at higher energy transfer. The first recoil peak corresponds to recoiling molecules undergoing a rotational transition from $J=0$ to the $J=1$ states. The additional recoil peaks can be attributed to the recoiling molecule undergoing higher order rotational transitions. At high energy transfer the roto-vibrational spectrum of $\mathrm{H}_{2}$ is superimposed over the recoil background [60].

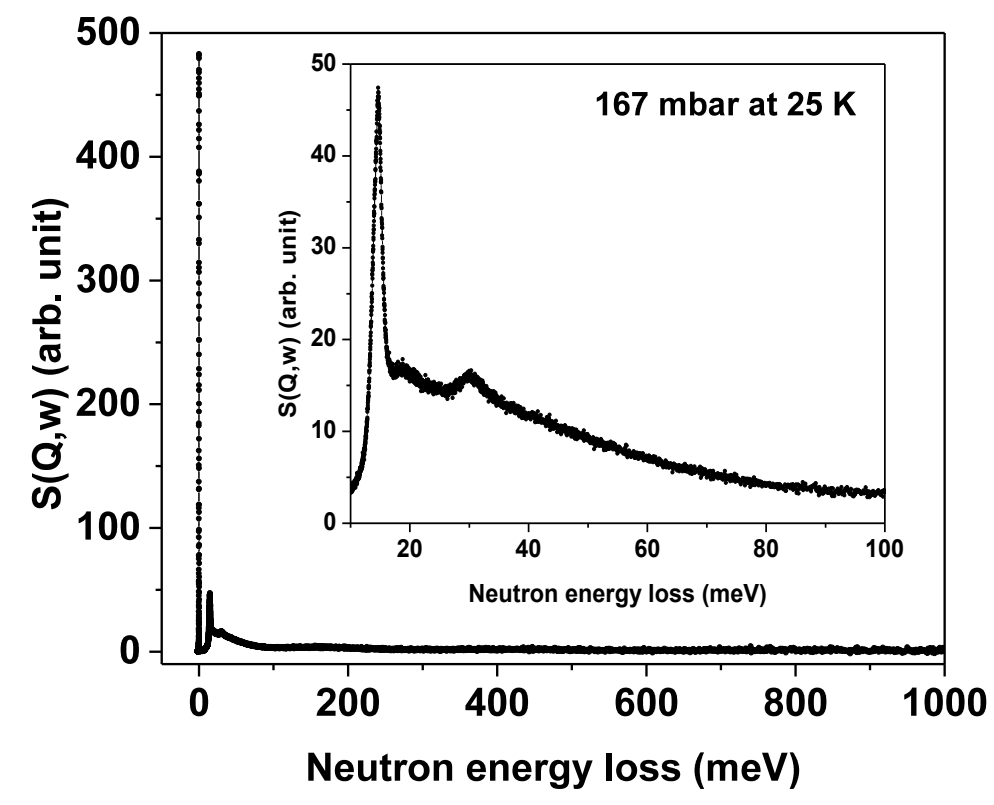

Figure 2. Typical INS spectrum for adsorbed $\mathrm{p}-\mathrm{H}_{2}$ at $25 \mathrm{~K}$ corresponding to $P=167$ mbar loading $\left(6.7 \mathrm{mmol} / \mathrm{g}, \theta_{P}=0.21\right)$. The zoomed view (inset) shows rotational and recoil peaks. $S(Q, w)$ is the dynamical structure factor in forward direction. 
Only the elastic scattering and first rotational peak are analyzed here. The integrated intensity of the INS spectrum is proportional to the total amount of $\mathrm{H}_{2}$ in the sample. Gas phase $\mathrm{H}_{2}$ molecules do not scatter elastically (they just recoil) so their contribution to the total spectra is smeared along the whole energy range. Elastic scattering occurs only from immobile (solid-like) $\mathrm{H}_{2}$ molecules, whereas partially mobile (liquid-like) $\mathrm{H}_{2}$ contributes towards the quasielastic broadening at energy transfers near $E=0$. The elastic and quasielastic scattering provide information on immobile and partially mobile o- $\mathrm{H}_{2}$ primarily, as the elastic scattering cross-section of $\mathrm{p}-\mathrm{H}_{2}$ is quite small [59]. The recoilless rotational peak can only be observed for immobile solid-like $\mathrm{H}_{2}$ molecules [40,41]. Thus, the integrated rotational peak area gives information on the immobile $\mathrm{p}-\mathrm{H}_{2}$ molecules. The shape and position of the rotational peak provides information about the interaction potential of the binding sites

\subsection{Surface Potential at Very Low Initial Loading}
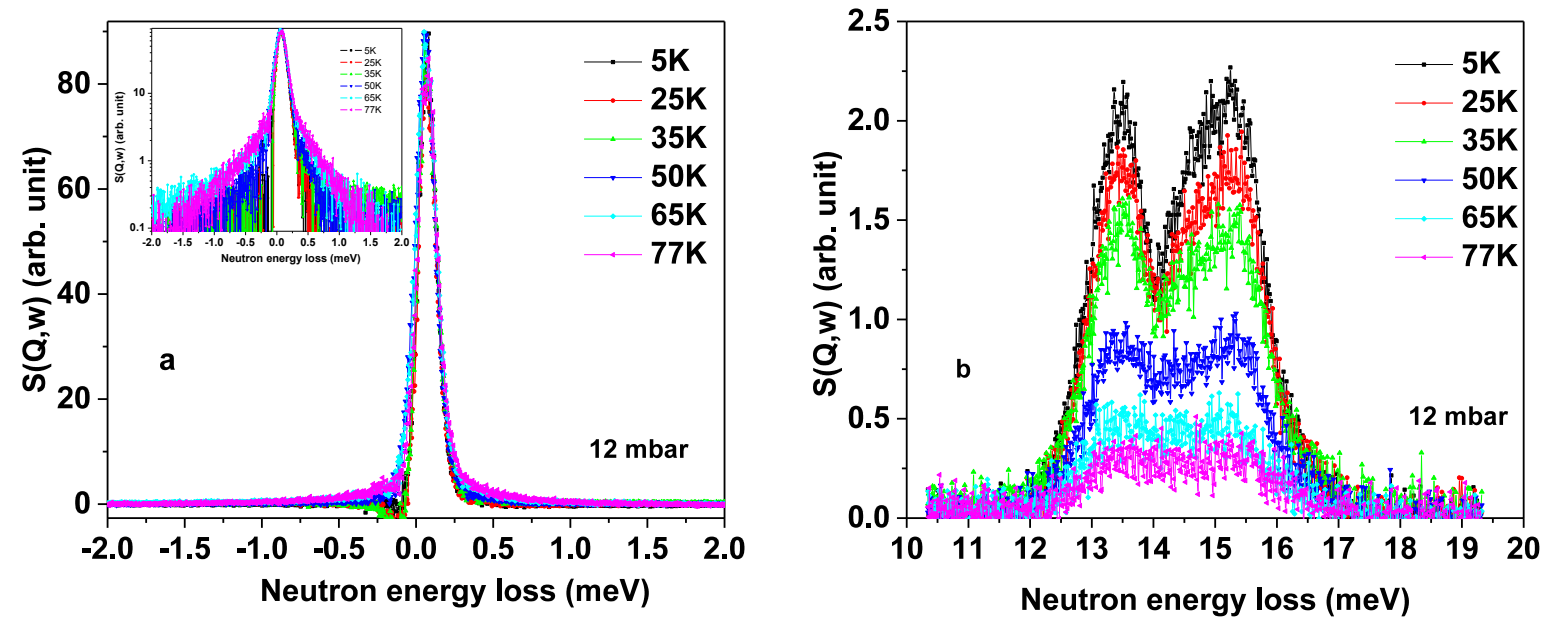

Figure 3. Elastic and quasielastic scattering signal (a) and rotor peak (b) at $P=12$ mbar loading $\left(1.69 \mathrm{mmol} / \mathrm{g} ; \theta_{P}=0.05\right)$ as a function of temperature. Inset in (a) shows data on the logarithmic scale. $S(Q, w)$ is the dynamical structure factor in forward direction. 
The background and baseline subtracted elastic/quasielastic scattering signal and rotor peak corresponding to the lowest dosing of $\mathrm{H}_{2}$ are shown in Figure 3. The method of baseline subtraction is shown in Figures S4 and S5 of Supplementary Information. At the lowest loading the quasielastic signal broadens with temperature. A strong splitting of the rotational peak is observed, indicating that rotational motion of low-dose adsorbed $\mathrm{H}_{2}$ is strongly hindered. The free quantum rotor energy for para $\rightarrow$ ortho transition in solid hydrogen is 14.7 $\mathrm{meV}$ [40]. The degeneracy of the ortho state is lifted in the presence of a strong anisotropic potential. The $J=1$ state is split into $m= \pm 1$ (rotation axis of molecule perpendicular to the adsorbed surface) and $m=0$ (rotation axis of molecule parallel to the adsorbed surface) [47]. The magnitude of rotor peak splitting depends on the anisotropy of the potential. The rotor peaks were fitted using two Gaussians as shown in Figure S6 of the Supplementary Information. The peak energies are $13.4( \pm 0.1) \mathrm{meV}$ and $15.1( \pm 0.1) \mathrm{meV}$. The $\Delta E_{\text {rot }}$ is calculated as $1.7 \mathrm{meV}$. Although peak intensity decreases with temperature, the peak positions and the magnitude of their splitting do not vary with temperature at this very low loading $\left(1.69 \mathrm{mmol} / \mathrm{g} ; \theta_{P}=0.05\right)$ corresponding to $P=12 \mathrm{mbar}$ exposure at $77 \mathrm{~K}$.

The splitting of the rotational peak can be understood as follows: The adsorption potential $V(z, \theta)$ of a molecule is primarily characterized by its well depth and anisotropy, where $z$ is the distance of the center of mass of the molecule from the surface and $\theta$ is the polar angle of the molecular axis with the surface normal [47]. The potential can be expressed as sum of Legendre functions, i.e., $V(z, \theta)=\sum_{l=0}^{l=\infty} V_{l}(z) P_{l}(\cos \theta)$. Since $\mathrm{H}_{2}$ is a homonuclear molecule, $l$ is restricted to even integers. For isotropic potential $V_{0}(z)$ the value of $l$ becomes 0 and the adsorbed molecules behave as free rotors. Furthermore, rotational transition is expected to occur at $14.7 \mathrm{meV}$ for $\mathrm{H}_{2}$ adsorbed under an isotropic potential.

The anisotropic potential can be expressed as $V(z, \theta)=V_{0}(z)+V_{2}(z) P_{2}(\cos \theta)$, where the ratio $V_{2} / V_{0}$ defines the degree of anisotropy. For $V_{2} / V_{\mathrm{o}}<<1$, a slight shift of the rotational line is 
observed. In this case, $\mathrm{H}_{2}$ molecules behave as nearly free rotors. However, for $V_{2} / V_{\mathrm{o}}<1$, the degeneracy of the $J=1$ state is removed and splitting of rotational line occurs. In this case, $\mathrm{H}_{2}$ molecules undergo hindered rotational motion. In order to estimate the rotational barrier, a double-well hindering adsorption potential of the form $V(z, \theta)=\frac{V_{2}}{2}(1-\cos 2 \theta)$ was assumed. The rotational constant $B$ and the potential barrier $V_{2}$ for the lowest $\mathrm{H}_{2}$ loading have been calculated using DAVE software [61]. The values found are $B=7.27 \mathrm{meV}$ and $V_{2}=$ $4.27 \mathrm{meV}$, respectively. In fact the value of $B$ determined here (7.27 instead of 7.35) can be attributed to the difference in the zero point energy between ortho- and para- hydrogen [62]. The strong anisotropic potential is located at low-symmetry sites, like surface steps and kinks. It was previously shown that wrinkling of the graphene sheet is driven by topological defects that are not well controlled during charring and activation of carbons [19]. The narrow spaces in micropores create a strongly anisotropic environment. The strongest adsorption energy sites, where adsorption occurs at very low loadings, are the narrowest pores with sub-nanometer size present in UMC. Here the overlapping potential from opposite pore walls enhances the confinement effect. The strong anisotropic potential experienced by $\mathrm{H}_{2}$ molecules confined in micropores causes high density of the adsorbed $\mathrm{H}_{2}$ as observed by SANS measurements [24]. 


\subsection{Effect of Pressure and Temperature at Low Surface Loading}
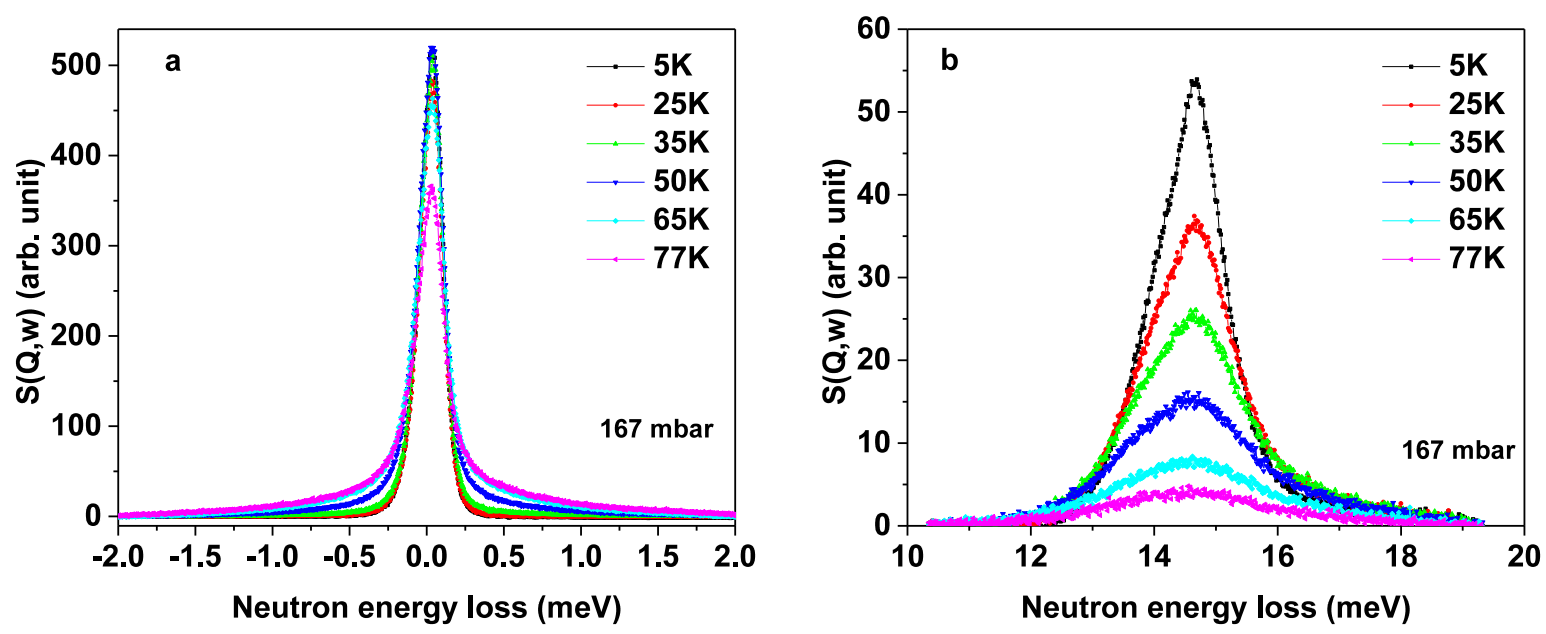

Figure 4. Elastic and quasielastic scattering signal (a) and rotor peak (b) at $P=167$ mbar loading $\left(6.73 \mathrm{mmol} / \mathrm{g} ; \theta_{P}=0.21\right)$ as a function of temperature.

The elastic/quasielastic scattering signal and rotor peak corresponding to exposure to $P=167$ mbar at $77 \mathrm{~K}\left(6.73 \mathrm{mmol} / \mathrm{g} ; \theta_{\boldsymbol{P}}=0.21\right)$ are shown in Figure 4. Compared to $12 \mathrm{mbar}$ loading $\left(\theta_{P}=0.05\right)$ a much more significant broadening of the quasielastic signal with temperature is observed at 167 mbar loading $\left(\theta_{\boldsymbol{P}}=0.21\right)$. This indicates that the mobility of adsorbed hydrogen is higher at this loading and that is has strong temperature dependence. It is important to recall that broadening of the quasielastic signal was much less pronounced at the lowest loading. This indicates that molecules occupy sites with relatively weaker adsorption potential as loading increases. It is also observed from Figure 4 that the rotor peak reduces to a single asymmetric peak at $14.6 \mathrm{meV}$, indicating nearly free quantum rotor behavior of $\mathrm{H}_{2}$. At this higher loading the strongest adsorption sites in narrow micropores are saturated, and adsorption occurs in larger pores, with reduced anisotropy in the adsorption potential. 
The INS spectra for intermediate loadings (52 mbar and $100 \mathrm{mbar}$ ) are shown in Figures S7 and S8 of Supplementary Information. As expected, the results for intermediate loadings are in between those obtained for the lowest and highest loadings. At $52 \mathrm{mbar}\left(3.65 \mathrm{mmol} / \mathrm{g} ; \theta_{P}\right.$ $=0.11)$ the quasielastic signal shows appreciable broadening at $77 \mathrm{~K}$, and the splitting of the rotor peak is less prominent than at the lowest loading. Similarly, the high temperature broadening of the quasielastic signal is also observed at $100 \mathrm{mbar}\left(5.14 \mathrm{mmol} / \mathrm{g} ; \theta_{P}=0.16\right)$, and the rotor peak looks similar to that at $167 \mathrm{mbar}\left(6.73 \mathrm{mmol} / \mathrm{g} ; \theta_{P}=0.21\right)$.
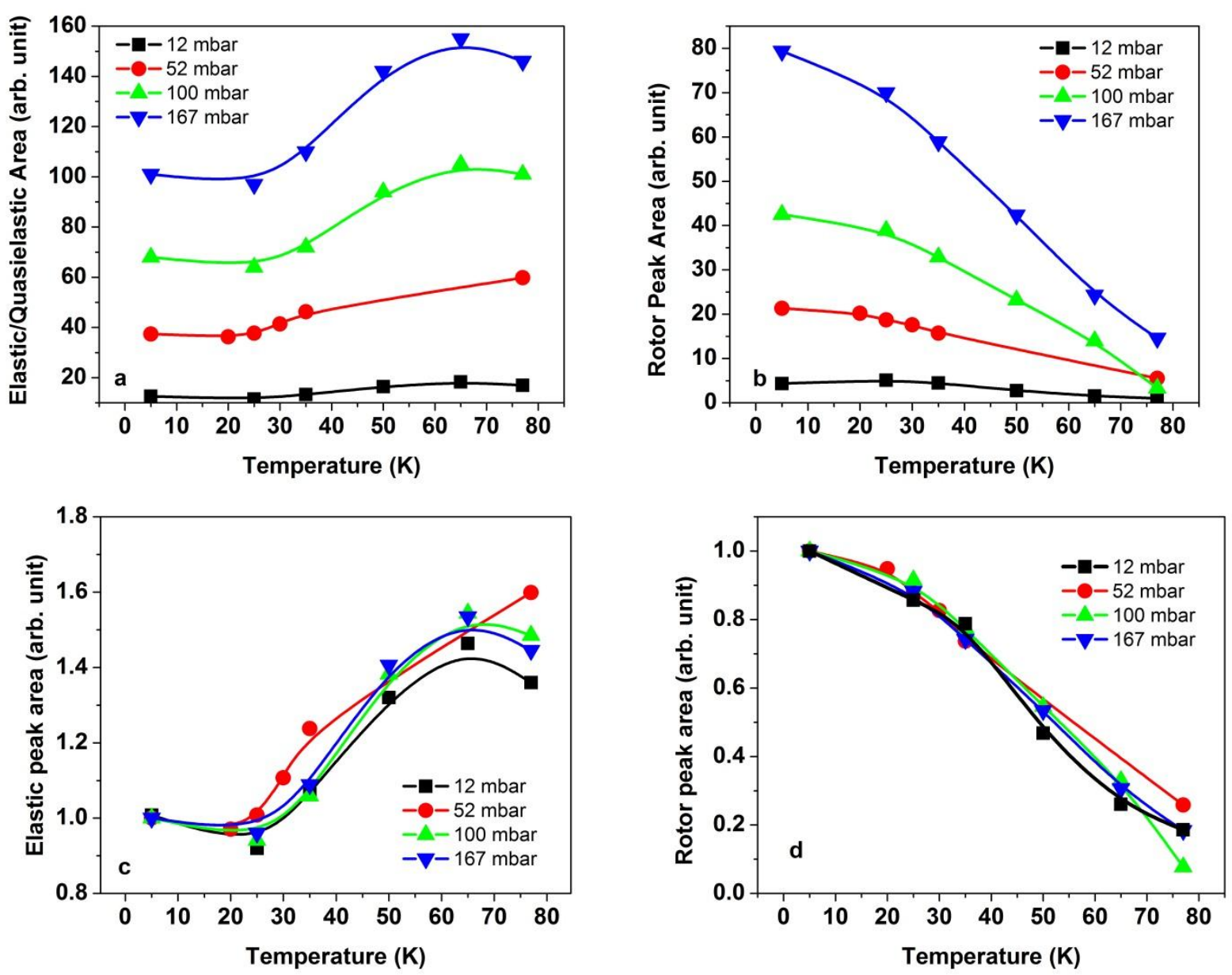

Figure 5. Integrated intensity of elastic/quasielastic signal (a) and rotor peak (b) as a function of temperature and loading. Data at each temperature were normalized to the respective integrated intensity at $5 \mathrm{~K}(\mathrm{c}, \mathrm{d})$. Error bars are less than the size of the symbols used. The lines are a guide to the eye. 
The integrated intensity of elastic/quasielastic signal and the rotor peak at each loading is shown in Figure 5 as a function of temperature. The intensity of the elastic/quasielastic signal remains approximately constant below the supercritical temperature of bulk $\mathrm{H}_{2}(33.14 \mathrm{~K})$ at all loadings. This indicates that the population of immobile and partially mobile $\mathrm{H}_{2}$ remains approximately constant up to about $33 \mathrm{~K}$. However, the elastic/quasielastic intensity increases at higher temperatures. Moreover, if the constant loading data from Figure 5a are normalized to the respective integrated intensities at $5 \mathrm{~K}$ they all collapse on the same overlapping trend (Fig. 5c). This indicates that the proportional effect of temperature is caused by thermally induced para-ortho conversion.

It is worth mentioning that a prior QENS investigation of $\mathrm{H}_{2}$ adsorbed on UMC showed that adsorbed $\mathrm{H}_{2}$ species become mobile between 19 and $22 \mathrm{~K}$ [32] when the effective molecular size $\left(\rho=\sigma_{o}+\lambda_{B}\right)$ of quantized light $\mathrm{H}_{2}$ molecules dropped to values comparable with the wall-to-wall width of prevalent micropores in UMC (about 6 A) [31]. Two factors determine the drop of $\rho$ on the increase of temperature in conditions when the quantum effects cannot be neglected: The thermal wave length (de Broglie) of light $\mathrm{H}_{2}$ molecules, $\lambda_{B}=h /\left(2 \pi m k_{B} T\right)^{1 / 2}$, drops between $5.5 \AA$ at $10 \mathrm{~K}$ to only $1.4 \AA$ at $77 \mathrm{~K}$. This variation also affects the $\mathrm{C}-\mathrm{H}_{2}$ interaction potential for quantized $\mathrm{H}_{2}$ molecules and their equilibrium separation distance $\sigma_{o}$ to the carbon surface. As a consequence, $\mathrm{H}_{2}$ is "bulkier" than $\mathrm{D}_{2}$ at cryogenic temperature, and moves slower on the increase of temperature, as proven by the delayed broadening of the quasielastic signal observed in QENS experiments [31]. Unfortunately that conclusion could not be strengthened by the results of the current experiment because the limited resolution of VISION spectrometer did not allow accurate analysis of the quasielastic signal broadening at such low temperatures. 
The rotor peak area in Figure 5b shows an interesting variation with the temperature: At all surface loadings investigated all normalized curves are overlapped (Fig. 5d). Up to $25 \mathrm{~K}$ the integrated peak intensity decreases by about $20 \%$ of its initial value at $5 \mathrm{~K}$. At higher temperatures the drop in rotor peak area is faster, about $60 \%$ of its initial value between 35 and $77 \mathrm{~K}$. The drop in intensity of the rotor peak on increase of temperature is most probably caused by thermal induced mobility (rather than thermal desorption) since its temperature variation is essentially independent on the equilibrium pressure. At the two lowest pressures of these experiments bulk $\mathrm{H}_{2}$ changes from solid directly to gas phase at about $13.7 \mathrm{~K}$ (Figure 1). At the two other pressures bulk $\mathrm{H}_{2}$ passes shortly through a liquid phase on increase of temperature The results in Figure 5 suggest that $\mathrm{H}_{2}$ hosted in UMC ultramicropores at low loadings $\left(0.05<\theta_{P}<0.2\right)$ has a different behavior. A rough analysis of pressure and temperature correlations at constant levels of rotor peak area (constant loading) using the Clausius-Clapeyron equation

$\ln \left(\frac{P_{1}}{P_{2}}\right)=-\frac{\Delta H}{R}\left(\frac{1}{T_{1}}-\frac{1}{T_{2}}\right)$

indicates that the process is endothermic $(\Delta H>0)$. This is expected for a solid-to-liquid phase change. The enthalpy change at high loading $\left(22.5 \mathrm{mmol} / \mathrm{g} ; \theta_{P}=0.7\right)$ is $\Delta H=0.134$ $\mathrm{kJ} / \mathrm{mol}$, close to the latent heat of fusion of bulk hydrogen $(0.116 \mathrm{~kJ} / \mathrm{mol})$, but smaller than the endothermic heat of para $\rightarrow$ ortho conversion $(1.062 \mathrm{~kJ} / \mathrm{mol}$ at $20 \mathrm{~K})$. The enthalpy change was therefore identified with the latent heat of fusion for solid-like hydrogen hosted in carbon pores. At high loading a large fraction of adsorbed hydrogen resides in larger pores, and resembles solid $\mathrm{H}_{2}$ at low temperatures. However, the enthalpy change increases as shown in Figure $6 \mathrm{a}$ at lower loadings, and reaches about $2.6 \mathrm{~kJ} / \mathrm{mol}$ at $3.7 \mathrm{mmol} / \mathrm{g}\left(\theta_{P}=0.1\right)$. At these low loading all hydrogen is trapped in ultramicropores and subject to a strongly anisotropic potential, as discussed above. 
Figure $6 \mathrm{~b}$ compares the rotor peak area measured at $77 \mathrm{~K}$ in all three series of experiments with the adsorption isotherm measured gravimetrically at the same temperature. The almost one-to-one agreement between the two was used to calibrate the arbitrary units used for integrated rotor peak area against the hydrogen loadings at $77 \mathrm{~K}$. Figure $6 \mathrm{a}$ indicates that at all pressures investigated, a certain fraction (constant) of the amount adsorbed is held in narrow pores and is thermally converted to $\mathrm{o}-\mathrm{H}_{2}$. Unfortunately the calibration of rotor peak areas cannot be used for quantitative evaluation of elastic and quasielastic components obtained by deconvolution of the scattering signal close to zero energy. One reason is the large cross section difference between $\mathrm{o}-\mathrm{H}_{2}$ species, which are responsible for the elastic and quasielastic components, and $\mathrm{p}-\mathrm{H}_{2}$ species, which are quantified by the rotor peak area. Another reason is the unknown spectrometer response to events separated by almost $15 \mathrm{meV}$ on the energy scale.
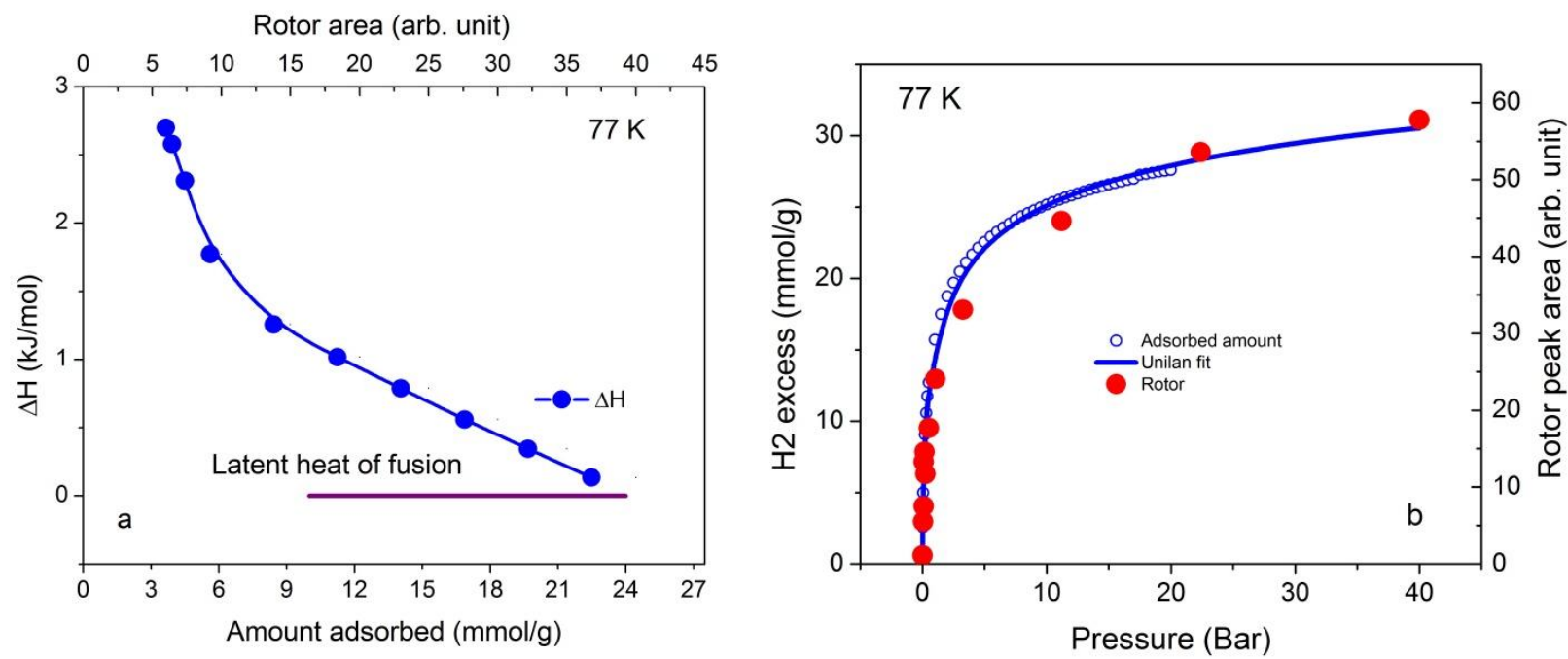

Figure 6: (a) Variation of fusion enthalpy of immobile $\mathrm{H}_{2}$ adsorbed on UMC as a function of surface loading; (b) Comparison of rotor peak area of immobile $\mathrm{H}_{2}$ at $77 \mathrm{~K}$ and the gravimetric amounts of adsorbed $\mathrm{H}_{2}$ at the same temperature. 


\subsection{High Pressure Effect at $77 \mathrm{~K}$}

Figure 7 shows the results of the second series of experiments, where the behavior of adsorbed $\mathrm{H}_{2}$ was examined from spectra recorded at $77 \mathrm{~K}$ and pressures between 0.1 and 40 bar. The background subtracted high pressure INS spectra are shown in Figure S9 of Supplementary Information. The quasielastic signal shows a visible increase with pressure. The strength of rotational peak also increases with pressure. For a quantitative analysis, the peak intensities were integrated between $-2 \mathrm{meV}$ and $2 \mathrm{meV}$ (quasielastic) and between 10 $\mathrm{meV}$ and $25 \mathrm{meV}$ (rotational peak). The baseline was subtracted from both the quasielastic signal and the rotor peak as shown in Figures S4 and S5 of the Supplementary Information.
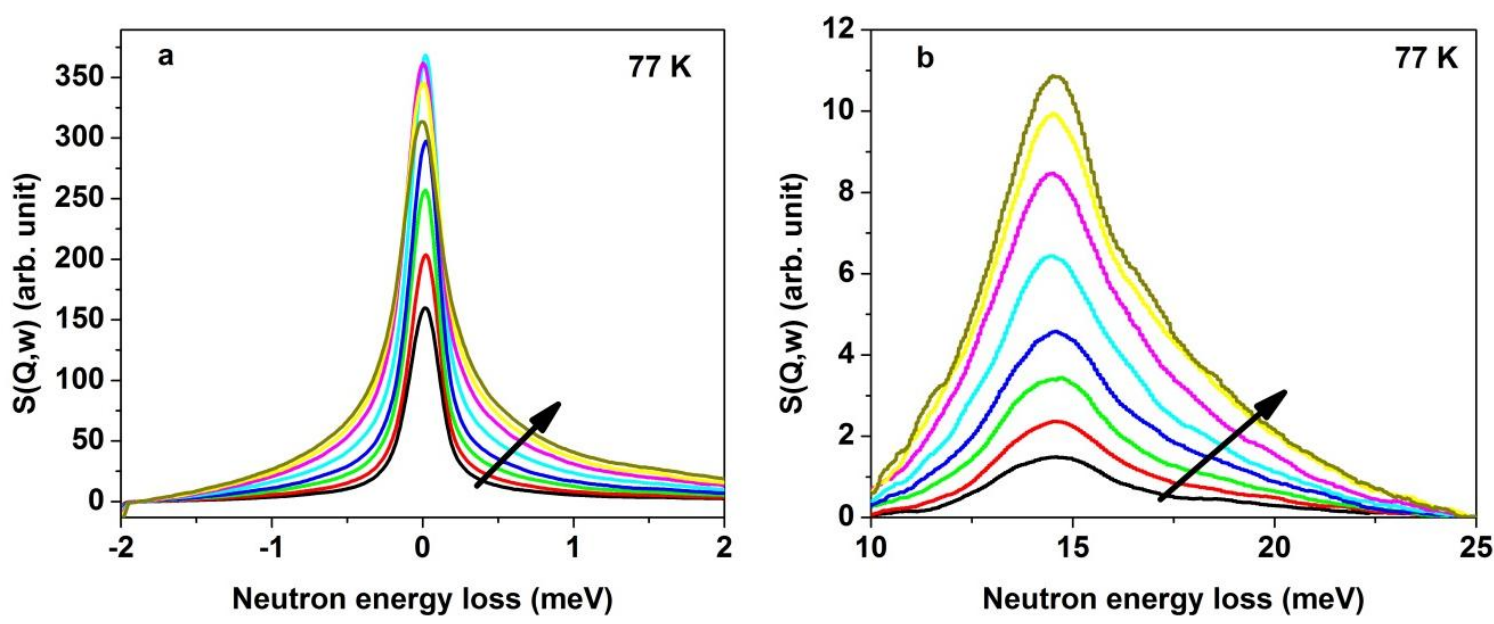

Figure 7 Evolution of elastic/quasielastic signal (a) and rotor peak (b) as a function of pressure $(0.10,0.23,0.51,1.03,3.25,11.2,22.4$ and 40.0 bar $)$ at $77 \mathrm{~K}$.

In order to distinguish between the contributions from the solid- and liquid-like hydrogen, the elastic and quasielastic scattering signals need to be separated. This can be done using the 
standard approach to analysis of quasielastic spectra, which is not normally performed at inelastic neutron spectrometers, but can be employed at VISION due to its sufficiently high energy resolution and intensity. The analysis involves fitting the data with a numerical convolution of the spectrometer resolution function, $R(w)$, with the model scattering function describing the diffusivity, $S(w)$, plus typically a linear fitted background. For the current system, the UMC sample measured prior to $\mathrm{H}_{2}$ dosing was used for the $R(\mathrm{w})$ because it had no associated quasielastic scattering. A Lorentzian function describing a diffusion process was used for the $S(w)$. The resulting model fitted to the experimental intensity was thus

$$
\begin{aligned}
& I(w)=C_{0}\left[x \delta(w)+(1-x) \frac{1}{\pi} \frac{\Gamma}{\Gamma^{2}+w^{2}}\right] \otimes R(w)+\left(C_{1} w+C_{2}\right)= \\
& C_{0}\left[x R(w)+(1-x) \frac{1}{\pi} \frac{\Gamma}{\Gamma^{2}+w^{2}} \otimes R(w)\right]+\left(C_{1} w+C_{2}\right)
\end{aligned}
$$

Here the parameter $x$ is the fraction of the elastic (vs. elastic plus quasielastic) scattering signal, which is associated with the solid-like immobile hydrogen. In turn, the fraction of quasielastic (vs. elastic plus quasielastic) scattering signal, (1-x), is associated with the liquidlike partially mobile hydrogen. It should be noted that subtraction of the background spectrum, the UMC sample measured prior to $\mathrm{H}_{2}$ dosing, from the fitted data is essential for accurate determination of the solid- and liquid-like $\mathrm{H}_{2}$ contributions. 


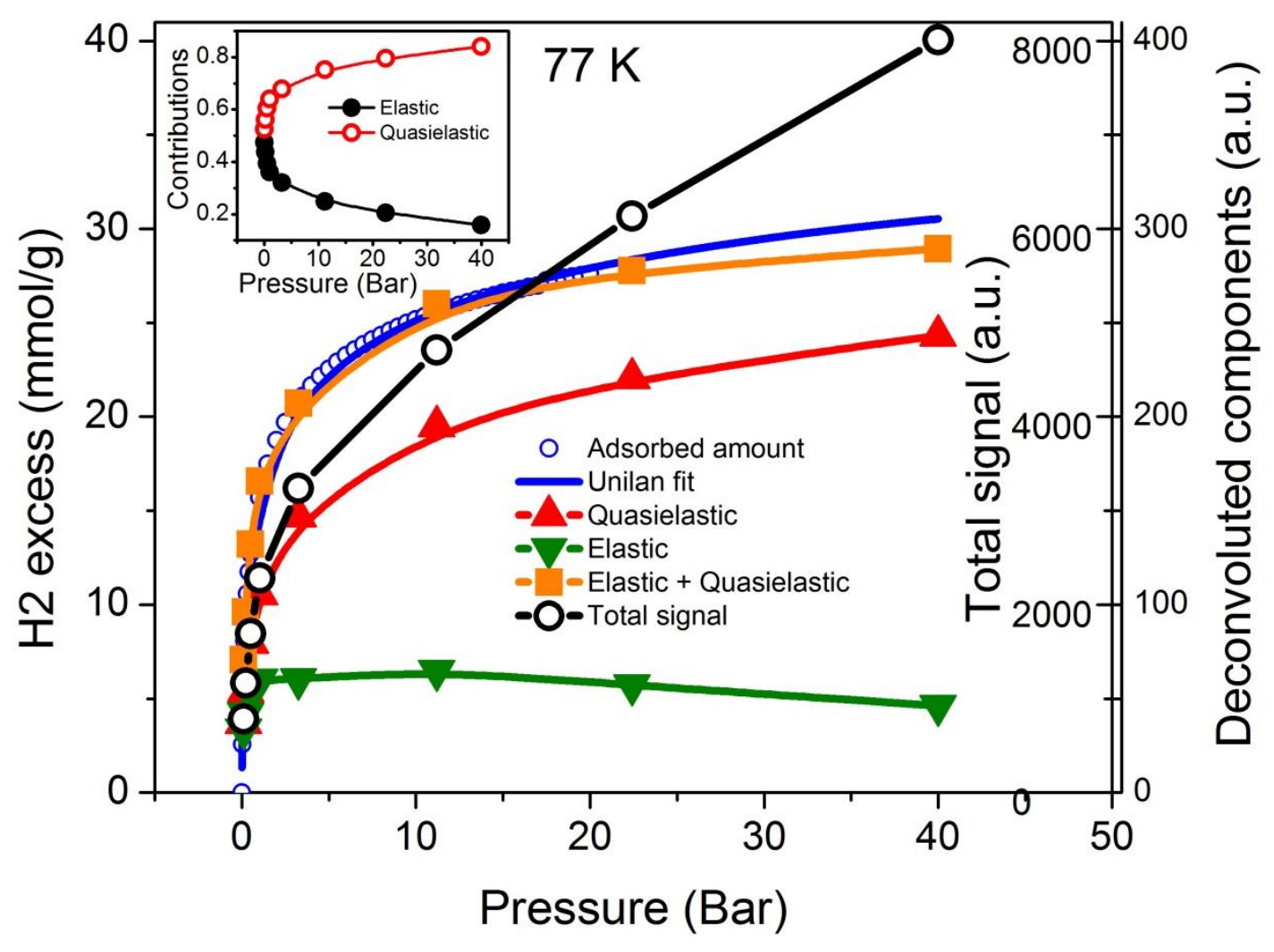

Figure 8. Comparison between $\mathrm{H}_{2}$ excess adsorption, deconvoluted elastic and quasielastic signals, and total INS signal as a function of pressure at $77 \mathrm{~K}$. Inset: Pressure effect on relative contributions of elastic and quasielastic signals.

Figure 8 shows the pressure dependence of integrated intensities obtained from deconvolution of elastic/quasielastic signals. Recall that the elastic and quasielastic signals originate, respectively, from the immobile (solid-like) and semi-mobile (liquid-like) hydrogen. Both signals increase sharply at pressures below about 1 bar, which is the range of initial micropore filling at $77 \mathrm{~K}$. Using the Unilan isotherm fit, one can estimate that equilibrium hydrogen adsorption at 1 bar at $77 \mathrm{~K}$ results in about $50 \%$ filling of total adsorption capacity. At these pressures adsorbed hydrogen exists both in solid- and liquidlike phase at $77 \mathrm{~K}$, as shown by the elastic and quasielastic signals. At pressures above 1 bar 
the elastic component remains (almost) constant and the growth of the quasielastic component bends slowly towards a saturation plateau at high pressures. Significantly, the sum of elastic and quasielastic signals follows consistently the adsorption isotherm measured gravimetrically (up to 20 bar) and the Unilan prediction (above 20 bar). The inset in Figure 8 shows the relative contributions of elastic and quasielastic signals. At very low pressure (100 mbar, $\left.\theta_{P}=0.2\right)$ the two components have almost equal contribution, but as the loading increases the balance shifts rapidly and the liquid-like $\mathrm{H}_{2}$ species dominate (75-80\%); The solid-like species still contribute about $20-25 \%$ of total adsorption up to the highest pressures. Obviously, $\mathrm{H}_{2}$ adsorbed at low loading is trapped first in ultramicropores $(<0.7$ $\mathrm{nm})$ where it remains immobile up to high temperatures. At higher loading adsorption continues in supermicropores $(0.7$ to $2 \mathrm{~nm})$ and then in lower mesopores (> $2 \mathrm{~nm})$, where a stable liquid-like phase was identified even at $77 \mathrm{~K}$ (Figure 8). The $20: 80$ ratio between elastic and quasielastic components reflects a similar ratio between the ultramicropores and the combined volumes of supermicropores and lower mesopores (see Figure S1 in Supplementary Information).

The secondary axis in Figure 8 shows the variation of the total signal intensity of the INS spectra integrated in the range of $-2 \mathrm{meV}$ to $1000 \mathrm{meV}$. The total intensity is proportional to total $\mathrm{H}_{2}$ population in the sample cell and includes free $\mathrm{H}_{2}$ gas along with adsorbed molecules, both partially mobile and immobile. Although the arbitrary units used for quantification of total intensity, elastic/quasielastic, rotor components are difficult to relate to each other, raw data (Figure 2) and area-integrated signals (Figure 8) show that the total signal intensity is much larger than the sum of elastic/quasielastic and rotor components. After an initial fast increase for pressures $<1$ bar, the total integrated signal varies linearly at higher pressures. The fast initial increase at low pressure is mainly contributed by adsorbed solid- and liquid-like $\mathrm{H}_{2}$ hosted in carbon. The linear increase at higher pressures reflects the 
addition of more $\mathrm{H}_{2}$ gas to the sample cell (free volume and UMC interparticle voids). The linear trend is expected from the equation of states of the free bulk gas.

\subsection{Temperature Effect at Constant Pressure}

The high temperature behavior of adsorbed $\mathrm{H}_{2}$ was further monitored in the third experiment. The INS spectra were recorded at constant pressure (3.25 bar) and variable temperature (77, 110 and $150 \mathrm{~K}$. The elastic/quasielastic signal at $150 \mathrm{~K}$ (Figure 9a) presents Fano-type resonance asymmetry. This is just due to slight over subtraction of the background signal; it is common in neutron scattering when two peaks slightly misalign and their relative intensities change. This particular case happens with the sample at $150 \mathrm{~K}$ (almost 5 times the critical temperature of molecular hydrogen) and the background measured at $77 \mathrm{~K}$. The background does not affect the shape of the peak at $110 \mathrm{~K}$, but it slightly over subtracts at $150 \mathrm{~K}$. The integrated intensity of the difference spectrum signal at $150 \mathrm{~K}$ is very small, so one cannot claim that there is appreciable elastic/quasielastic signal at this temperature. No immobile or partially mobile $\mathrm{H}_{2}$ exists at this temperature.
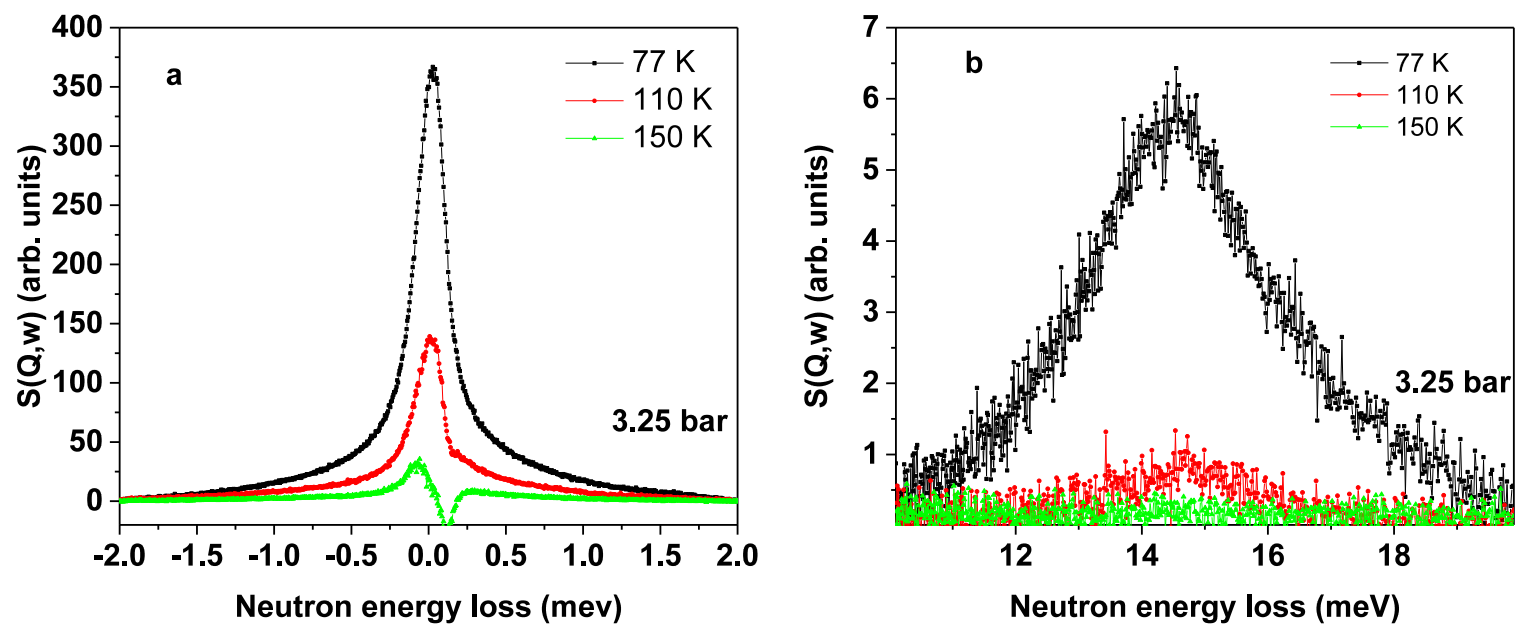
Figure 9. Elastic/quasielastic signal (a) and rotor peak (b) at 3.25 bar at high temperatures. Well-defined elastic/quasielastic and rotor peak exists up to $110 \mathrm{~K}$.

However, the elastic/quasielastic signal from immobile and partially mobile adsorbed $\mathrm{H}_{2}$ is clearly observed at 77 and $110 \mathrm{~K}$, well above the pressure-corrected boiling temperature of free gas $(25 \mathrm{~K})$ at 3.25 bar. Moreover, the rotor peak (solid-like, immobile $\mathrm{H}_{2}$ ) is visible at 77 $\mathrm{K}$ and even at $110 \mathrm{~K}$. This fact indicates that liquid- and solid-like hydrogen subsists in UMC pore systems at temperatures much higher than the critical point of bulk $\mathrm{H}_{2}(33.14 \mathrm{~K})$. The two peaks vanish at $150 \mathrm{~K}$ indicating that hydrogen was desorbed.

\subsection{The density of adsorbed hydrogen}

Figure S1 of Supplementary Information shows porosity distribution in UMC material, both in terms of pore volume and in terms of internal pore surface variation with the wall-to-wall pore width in slit shaped pores. This is the effective pore width available to hosted molecules. The porosity distribution was then correlated with the Unilan fitting of excess adsorption isotherms (Figure S2) at $77 \mathrm{~K}$ in an attempt to estimate the relationship between the amount adsorbed ( $\mathrm{mmol} / \mathrm{g})$ at every equilibrium pressure and the corresponding pore size (or volume) available for hosting the adsorbed phase. Using eq. (S2) from Supplementary Information, the surface area occupied by the adsorbate $\left(S_{P}\right)$ was estimated at each equilibrium pressure. Then the porosity distributions for internal pore surfaces, $S=S(d)$, and pore volumes, $V=$ $V(d)$, shown in Fig. S1 were correlated to determine the maximum pore width $d_{P}$ and the corresponding pore volume, $V_{P}$, that contains the adsorbed phase at every equilibrium pressure $P$. The implicit assumption of this procedure is that, on a patched surface consisting of a multitude of energetically uniform domains, different groups of sites are occupied in the 
decreasing order of their adsorption potential, which follows the increasing order of corresponding pore widths. Lastly, the equilibrium amounts adsorbed at every pressure, $q_{P}$, were correlated with the corresponding pore volumes used at that pressure, $V_{P}$, to estimate the effective density of the adsorbed phase hosted in pores of size $d_{P}$.

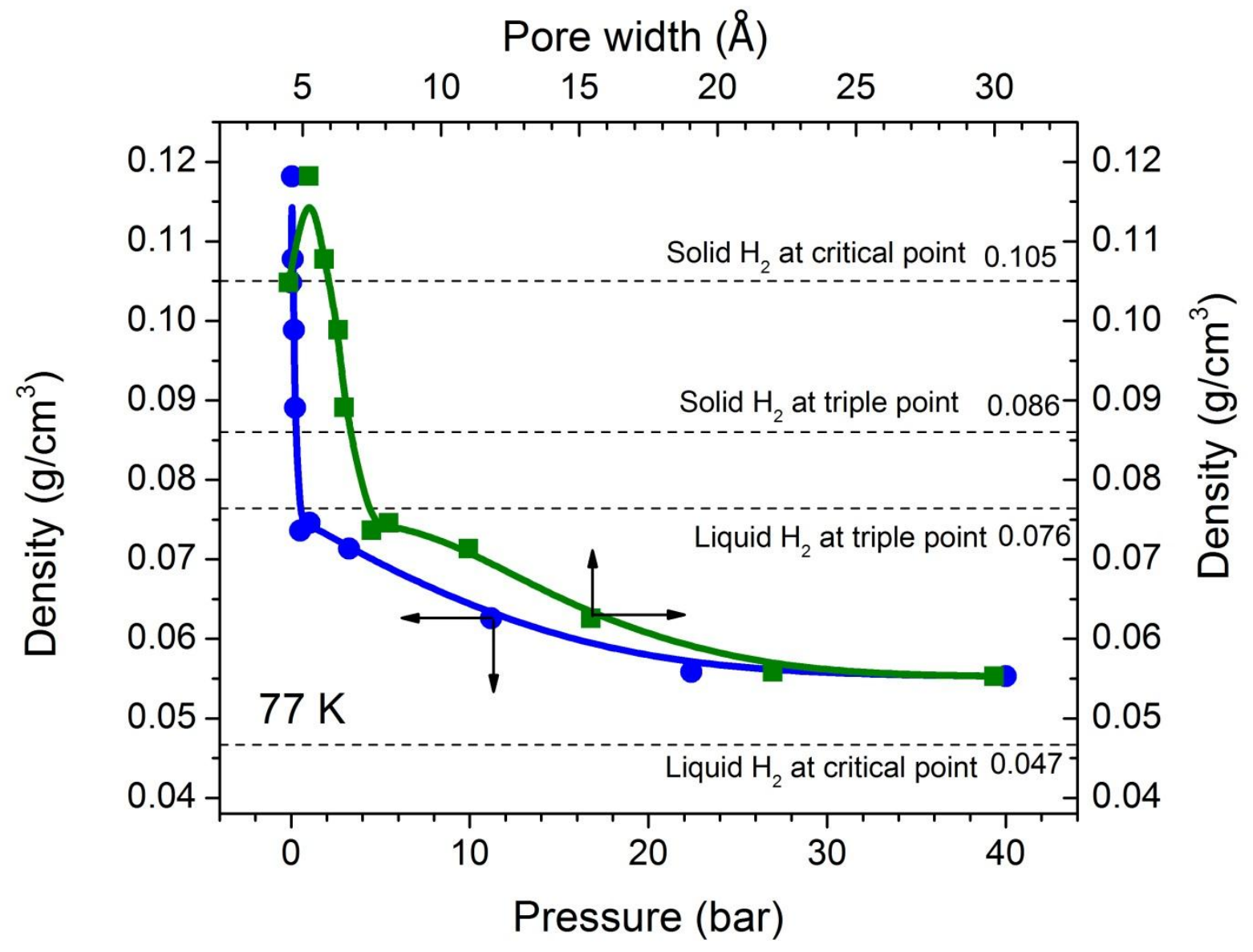

Figure 10: Effective hydrogen density in pores of various sizes at $77 \mathrm{~K}$ as a function of equilibrium pressure and pore width. The source for solid and liquid bulk $\mathrm{H}_{2}$ density is [37].

The results (Figure 10) show that the effective density of adsorbed hydrogen decreases with the increase of pore width and with the increase of pressure. For comparison, the density of bulk solid $\mathrm{H}_{2}$ and liquid $\mathrm{H}_{2}$ at triple point and critical point are marked by horizontal lines in Figure 10. These plots support the conclusion of this neutron scattering study: At $77 \mathrm{~K}$ and 
pressures below 1 bar the effective density of hydrogen in ultramicropores is comparable to that of solid $\mathrm{H}_{2}$. In this range of low loadings (experiments from series 1 and low pressure data from series 2) the adsorbed phase experience high densification in ultramicropores, as theoretically predicted $[63,64]$ and inferred from experiments [65] by others. The pressure range where solid-like density predicted by this estimation drops rapidly coincides with the initial pressure range where sharp drop was seen in the elastic contribution to total neutrons scattering (inset in Fig. 8).

Data at $77 \mathrm{~K}$ from the second (and third) series of experiments show a different behavior. Starting from pressures above 5 bar the estimated effective density of adsorbed hydrogen matches the density of liquid $\mathrm{H}_{2}$ and drops slowly at the increase of external pressure. At these pressures $\mathrm{H}_{2}$ is hosted in gradually larger pores that, along with ultramicropores, also include supermicropores and (at the highest pressures) the narrow mesopores. Fig. 8 shows that the surplus of adsorbed $\mathrm{H}_{2}$ condenses in liquid-like phase, which is now dominant, but a solid-like phase subsists at $77 \mathrm{~K}$ up to the highest pressure. The effective density is therefore an average value over pores of various sizes.

\section{Conclusion}

The main finding of this investigation is the persistence of solid-like (immobile) and liquidlike (partially mobile) $\mathrm{H}_{2}$ confined in carbon pores at temperatures and pressures remote from the critical point of free $\mathrm{H}_{2}$ gas. The presence of high-density, mobility-restricted phases (liquid-like and solid-like) at supercritical conditions is a general behavior of $\mathrm{H}_{2}$ confined in carbon nanopores. Solid-like and liquid-like $\mathrm{H}_{2}$ subsist up to 40 bar at $77 \mathrm{~K}$ and their ratio reflects the pore structure of the adsorbent. They diminish by desorption at higher 
temperatures, as expected, but solid-like $\mathrm{H}_{2}$ is present even at $110 \mathrm{~K}$ and 3.25 bar pressure, in conditions where bulk hydrogen exists only in gas form.

At the start of this study, the expected main output of VISION spectrometer, a state-of-the-art instrument optimized for vibrational spectroscopy, was the qualitative exploration of phase behavior of confined $\mathrm{H}_{2}$ as function of loading, temperature and pressure. However, later it became clear that more information could be extracted from this spectrometer. Thus, analysis of rotor peaks showed that the strongly anisotropic adsorption potential at the strongest adsorption sites (low coverage) becomes gradually more isotropic at higher coverages (weaker sites). Analysis of the broadened signal near zero energy transfer and deconvolution of the quasielastic component from the elastic component could also be performed, albeit within some instrument limitations. This analysis by itself pushes the limits of what was previously thought to be possible in vibrational indirect geometry spectrometers optimized for inelastic energy transfers commensurate with molecular vibrations. The relative proportion of mobile and immobile $\mathrm{H}_{2}$ species as a function of surface loadings, estimated from this analysis, revealed strong correlations with the pore structure of the carbon support. However, the line broadening of the quasielastic component as a function of temperature, and its Q dependence, could not be analyzed during these experiments. Such analysis would have been indeed relevant for determination in minute details of the diffusion mechanism.

In conclusion, the significance of present results reaches beyond the immediate interest in adsorption theory and confined fluids behavior. They may infuse in new impetus onto the search for the optimal hydrogen storage material, and underline the need of further efforts for developing ultramicroporous carbon materials with large volumes of narrowly distributed pores sizes, preferably close to the sub-nanometer size of hydrogen molecules. 


\section{Acknowledgements}

The research at ORNL's Spallation Neutron Source was supported by the Scientific User Facilities Division, Office of Basic Energy Sciences, U. S. Department of Energy. This research was supported in part by the ORNL Postdoctoral Research Associates Program, administered jointly by the ORNL and the Oak Ridge Institute for Science and Education. CIC and NCG acknowledge support from the Materials Science and Engineering Division, Office of Basic Energy Sciences, U.S. Department of Energy. The authors thank one anonymous reviewer for constructive queries and suggestions that helped raised the quality of the manuscript. This work was enthusiastically supported by the late Dr. Yuri Melnichenko, who unfortunately did not live to see the results.

\section{References}

[1] Dillon AC, Jones KM, Bekkedahi TA, Kiang CH, Bethune DS, Heben MJ. Storage of hydrogen in single-walled carbon nanotubes. Nature. 1997;386:377-9.

[2] Rosi NL, Eckert J, Eddaoudi M, Vodak DT, Kim J, O'Keeffe M, et al. Hydrogen storage in microporous metal-organic frameworks. Science. 2003;300:1127-9.

[3] Schlapbach L. Hydrogen-storage materials for mobile applications. Nature. 2001;414:353-8.

[4] Wang H, Gao Q, Hu J. High hydrogen storage capacity of porous carbons prepared by using activated carbon. J Am Chem Soc. 2009;131:7016-22.

[5] Xia Y, S.Walker G, Grant DM, R.Mokaya. Hydrogen storage in high surface area carbons: experimental demonstration of the effects of nitrogen doping. J Am Chem Soc. 2009;131:16493-9.

[6] Gogotsi Y, Dash RK, Yushin G, Yildirim T, Laudisio G, Fischer JE. Tailoring of Nanoscale Porosity in Carbide-Derived Carbons for Hydrogen Storage. J Am Chem Soc. 2005; 127:16006-7. 
[7] Gogotsi Y, Nikitin A, Ye HH, Zhou W, Fischer JE, Yi B, et al. Nanoporous carbidederived carbon with tunable pore size. Nat Mater. 2003;2:591 - 4.

[8] Gogotsi Y, Portet C, Osswald S, Simmons JM, Yildirim T, Laudisio G, et al. Importance of pore size in high-pressure hydrogen storage by porous carbons. Int $\mathrm{J}$ HydrogenEnerg. 2009;34:6314-9.

[9] Laudisio G, Dash RK, Singer JP, G.Yushin, Gogotsi Y, Fischer JE. Carbide-derived carbons: a comparative study of porosity based on small-angle scattering and adsorption isotherms. Langmuir. 2006;22:8945-50.

[10] Masika E, Mokaya R. Hydrogen storage in high surface area carbons with identical surface areas but different pore sizes: direct demonstration of the effects of pore size. J Phys Chem C. 2012;116:25734-40.

[11] Sevilla M, Mokaya R, Fuertes AB. High density hydrogen storage in superactivated carbons from hydrothermally carbonized renewable organic materials. Energ EnvironSci. 2011;4:1400-10.

[12] Texier-Mandoki N, Dentzer J, Piquero T, Saadallah S, P. D, Vix-Guterl C. Hydrogen storage in activated carbon materials: role of the nanoporous texture. Carbon. 2004;42:274451 .

[13] Yang ZX, Xia YD, Mokaya R. Enhanced hydrogen storage capacity of high surface area zeolite-like carbon materials. J Am Chem Soc. 2007;129:1673-9.

[14] Yushin G, Dash R, Jagiello J, Fischer JE, Gogotsi Y. Carbide-Derived Carbons: Effect of Pore Size on Hydrogen Uptake and Heat of Adsorption. Adv Funct Mater. 2006;16:2288-93.

[15] Aga RC, Fu CL, Krcmar M, Morris JR. Theoretical investigation of the effect of graphite interlayer spacing on hydrogen absorption. Phys Rev B. 2007;76:165404.

[16] Cabria I, Lopez MJ, Alonso JA. The optimum average nanopore size for hydrogen storage in carbon nanoporous materials. Carbon. 2007;45:2649-58.

[17] Marsh H, Rodríguez-Reinoso F. Activated carbon. 1 ed. Boston: Elsevier, Amsterdam 2006.

[18] Yurum Y, Taralp A, Veziroglu TN. Storage of hydrogen in nanostructured carbon materials. Int J Hydrogen Energ. 2009;34:3784-98.

[19] Guo J, Morris JR, Ihm Y, Contescu CI, Gallego NC, Duscher G, et al. Topological defects: origin of nanopores and enhanced adsorption performance in nanoporous carbon. Small. 2012;8:3283-8.

[20] Morris JR, Contescu CI, Chisholm MF, Cooper VR, Guo J, He L, et al. Modern approaches to studying gas adsorption in nanoporous carbons. J Mater Chem A. 2013;1: 9341-50.

[21] Strobel R, Garche J, Moseley PT, Jorissen L, Wolf G. Hydrogen storage by carbon materials. J Power Sources. 2006;159:781-801. 
[22] Bhat VV, Contescu CI, Gallego NC, Baker SF. Atypical hydrogen uptake on chemically-activated, ultramicroporous carbon. Carbon. 2010;48:1331-40.

[23] P Mitchell, P. C. H., Parker, S. F., Ramirez-Cuesta, A. J. \& Tomkinson, J. Vibrational spectroscopy with neutrons: with applications in chemistry, biology, materials science and catalysis. World Scientific (2005)

[24] Gallego NC, He L, Saha D, Contescu CI, Melnichenko YB. Hydrogen confinement in carbon nanopores: extreme densification at ambient temperature. J Am Chem Soc.

2011;133:13794-7.

[25] He L, Melnichenko YB, Gallego NC, Contescu CI, Guo J, Bahadur J. Investigation of morphology and hydrogen adsorption capacity of disordered carbons. Carbon. 2014;80:8290.

[26] Tanaka H, Kanoh H, El-Meraoui M, Steele AA, Yudasaka M, Ijima S, et al. Quantum effects on hydrogen adsorption in internal nanospaces of single-wall carbon nanohorns. $\mathrm{J}$ Phys Chem B. 2004;108:17457-65.

[27] Minuto FD, Policicchio A, Aloise A, G. AR. Liquid-like hydrogen in the micropores of commercial activated carbons. Intl J Hydrogen Energy. 2015;40:14562-72.

[28] Patchhkovskii S, Tse JS, Yurchenko SN, Zhechkov L, Heine T, Seifert G. Graphene nanostructures as tunable storage media for molecular hydrogen. Proceedings of the National Academy of Sciences. 2005;102:10439-44.

[29] Peng L, Morris JR. Prediction of hydrogen adsorption properties in expanded graphite model and in nanoporous carbon. J Phys Chem C. 2010;114:15522-9.

[30] Ting VP, Ramirez-Cuesta AJ, Bimbo N, Sharpe JE, Noguera-Diaz A, Presser V, et al. Direct evidence for solid-like hydrogen in a nanoporous carbon hydrogen storage material at supercritical temperatures. ACS Nano. 2015;9:8249-54.

[31] Contescu CI, Zhang H, Olsen RJ, Mamontov E, Morris JR, Gallego NC. Isotope Effect on Adsorbed Quantum Phases: Diffusion of $\mathrm{H}_{2}$ and $\mathrm{D}_{2}$ in Nanoporous Carbon. Phys Rev Lett. 2013;110:236102.

[32] Contescu CI, Saha D, Gallego NC, Mamontov E, Kolesnikov AI, Bhat VV. Restricted dynamics of molecular hydrogen confined in activated carbon nanopores. Carbon.

2012;50:1071-82.

[33] Baker FS, Beckler RK, Miller JR, Yan ZQ. Highly microporous carbons and process of manufacture. US patent. 1999;5965483.

[34] Bahadur J, Melnichenko YB, He L, Contescu CI, Gallego NC, Carmichael JR. SANS investigations of $\mathrm{CO}_{2}$ adsorption in microporous carbon. Carbon. 2015;95:535-44.

[35] Do, Duong D, "Adsorption Analysis: Equilibrium and Kinetics", Series on Chemical Engineering, vol. 2, Imperial College Press, 1998, p. 70-76.

[36] Seeger PA, Daemen LL, Larese JZ. Resolution of VISION, a crystal-analyzer spectrometer. Nuclear Instrum Methods Phys ResA. 2009;604:719-28. 
[37] Younglove BA, Thermophysical propertioes of fluids, I Argon, ethylene, parahydrogen, nitrogen, nitrogentrifluoride, and oxygen. J. Phys. Chem. Reference Data. 1982; Suppl. No. 1.

[38] Stoicheff BP. High resolution raman spectroscopy of gases: Ix. Spectra of $\mathrm{H}_{2}, \mathrm{HD}$, and $\mathrm{D}_{2}$. Can J Phys. 1957;35: 730-41.

[39] Silvera IF. The solid molecular hydrogens in the condensed phase: Fundamentals and static properties. Rev Mod Phys. 1980;52:393-452.

[40] Nielsen M. Phonons in solid hydrogen and deuterium studied by inelastic coherent neutron scattering. Phys Rev B. 1973;7:1626.

[41] Nielsen $\mathrm{M}$, Ellensen $\mathrm{W} . \mathrm{H}_{2}$ and $\mathrm{D}_{2}$ on graphite studied by neutron scattering. Fourteenth International Conference on Low Temperature Physics; 1975; Otaniemi, Finland: North-Holland, Amsterdam; 1975. p. 437.

[42] Mattera L, Rosatelli F, Salvo C, Tommasini F, Valbusa U, Vidali G. Selective adsorption of ${ }^{1} \mathrm{H}_{2}$ and ${ }^{2} \mathrm{H}_{2}$ on the (0001) graphite surface. Surface Science. 1980;93(2):51525 .

[43] Christmann K. Interaction of hydrogen with solid surfaces. Surf Sci Rep. 1988;9:1163.

[44] Vidali G, Ihm G, Kim H-Y, Cole MW. Potentials of physical adsorption. Surf Sci Rep. 1991;12:135-81.

[45] Brown CM, Yildirim T, Neumann DA, Heben MJ, Gennett T, Dillon AC, et al. Quantum rotation of hydrogen in single-wall carbon nanotubes. Chem Phys Lett. 2000;329:311-6.

[46] Georgiev PA, Ross DK, Albers P, Ramirez-Cuesta AJ. The rotational and translational dynamics of molecular hydrogen physisorbed in activated carbon: a direct probe of microporosity and hydrogen storage performance. Carbon. 2006;44:2724-38.

[47] Fukutani K, Sugimoto T. Physisorption and ortho-para conversion of molecular hydrogen on solid surfaces. Prog Surf Sci. 2013;88:279-348.

[48] Sugimoto T, Fukutani K. Effects of Rotational-Symmetry Breaking on Physisorption of Ortho-and Para- $\mathrm{H}_{2}$ on Ag (111). Phys Rev Lett. 2014;112:146101.

[49] Ramirez-Cuesta AJ, Jones MO, David WIF. Neutron scattering and hydrogen storage. Materials Today. 2009;12:54-61.

[50] Fernandez-Alonso F, Bermejo FJ, Cabrillo C, Loutfy RO, Leon V, Saboungi ML. Nature of the Bound States of Molecular Hydrogen in Carbon Nanohorns. Physical Review Letters. 2007;98(21):215503.

[51] Georgiev PA, Ross DK, De Monte A, Montaretto-Marullo U, Edwards RAH, Ramirez-Cuesta AJ, et al. In situ inelastic neutron scattering studies of the rotational and translational dynamics of molecular hydrogen adsorbed in single-wall carbon nanotubes (SWNTs). Carbon. 2005;43:895-906. 
[52] Georgiev PA, Ross DK, De Monte A, Montaretto-Marullo U, Edwards RAH, Ramirez-Cuesta AJ, et al. Hydrogen site occupancies in single-walled carbon nanotubes studied by inelastic neutron scattering. J PhysCondens Matter. 2004;16:L73-L8.

[53] Horsewill AJ, Goh K, Rols S, Ollivier J, Johnson MR, Levitt MH, et al. Quantum rotation and translation of hydrogen molecules encapsulated inside C60: temperature dependence of inelastic neutron scattering spectra. Philos Trans R Soc London, Ser A. 2013;371:20110627.

[51] Narehood DG, Kostov MK, Eklund PC, Cole MW, Sokol PE. Deep inelastic neutron scattering of $\mathrm{H}_{2}$ in single-walled carbon nanotubes. Phys Rev B. 2002;65:233401.

[55] Narehood DG, Pearce JV, Eklund PC, Sokol PE, Lechner RE, Pieper J, et al. Diffusion of $\mathrm{H}_{2}$ adsorbed on single-walled carbon nanotubes. Phys Rev B. 2003;67:205409.

[56] Ren Y, Price D. Neutron scattering study of $\mathrm{H}_{2}$ adsorption in single-walled carbon nanotubes. Appl Phys Lett. 2001;79:3684.

[57] Schimmel HG, Kearley GJ, Nijkamp MG, Visser CT, de Jong KP, Mulder FM. Hydrogen adsorption in carbon nanostructures: comparison of nanotubes, fibers, and coals. Chem - Eur J. 2003;9:4764-70.

[58] Young JA, Koppel JU. Slow neutron scattering by molecular hydrogen and deuterium. Phys Rev. 1964;135:A603-A11.

[59] Horsewill AJ, Panesar KS, Rols S, Ollivier J, Johnson MR, Carravetta M, et al. Inelastic neutron scattering investigations of the quantum molecular dynamics of a $\mathrm{H}_{2}$ molecule entrapped inside a fullerene cage. Physical Review B. 2012;85(20):205440.

[60] Olsen RJ, Beckner M, Stone MB, Pfeifer P, Wexler C, Taub H. Quantum excitation spectrum of hydrogen adsorbed in nanoporous carbons observed by inelastic neutron scattering. Carbon. 2013;58:46-58.

[61] Azuah RT, Kneller LR, Qiu Y, Tregenna-Piggott PWL, Brown CM, Copley JRD, et al. DAVE: a comprehensive software suite for the reduction, visualization, and analysis of low energy neutron spectroscopic data. J Res Natl InstStand Technol. 2009;114:341-58.

[62] FitzGerald SA, Yildirim T, Santodonato LJ, Neumann DA, Copley JRD, Rush JJ, et al. Quantum dynamics of interstitial $\mathrm{H}_{2}$ in solid $\mathrm{C}_{60}$. Physical Review B. 1999;60(9):6439-51.

[63] Kowalczyk P, Gauden PA, Terzyk AP, Bhatia SK, Thermodynamics of hydrogen adsorption in slit-like carbon nanopores at $77 \mathrm{~K}$. Classical versus path-integral Monte Carlo simulations, Langmuir. 2007; 23: 3666-72.

[64] Peng L, Morris JR. Prediction of hydrogen adsorption properties in expanded graphite model and in nanoporous carbon. JPhysChem. C. 2010; 114: 115522-29.

[64] Tanaka H, Kanoh H, El-Merraoui M, Steele WA, Yudasaka M, Iijima S, Kaneko K. Quantum effects in hydrogen adsorption in internal nanospaces of single-wall carbon nanohorns, JPhysChem. B. 2004; 108: 17457-65. 

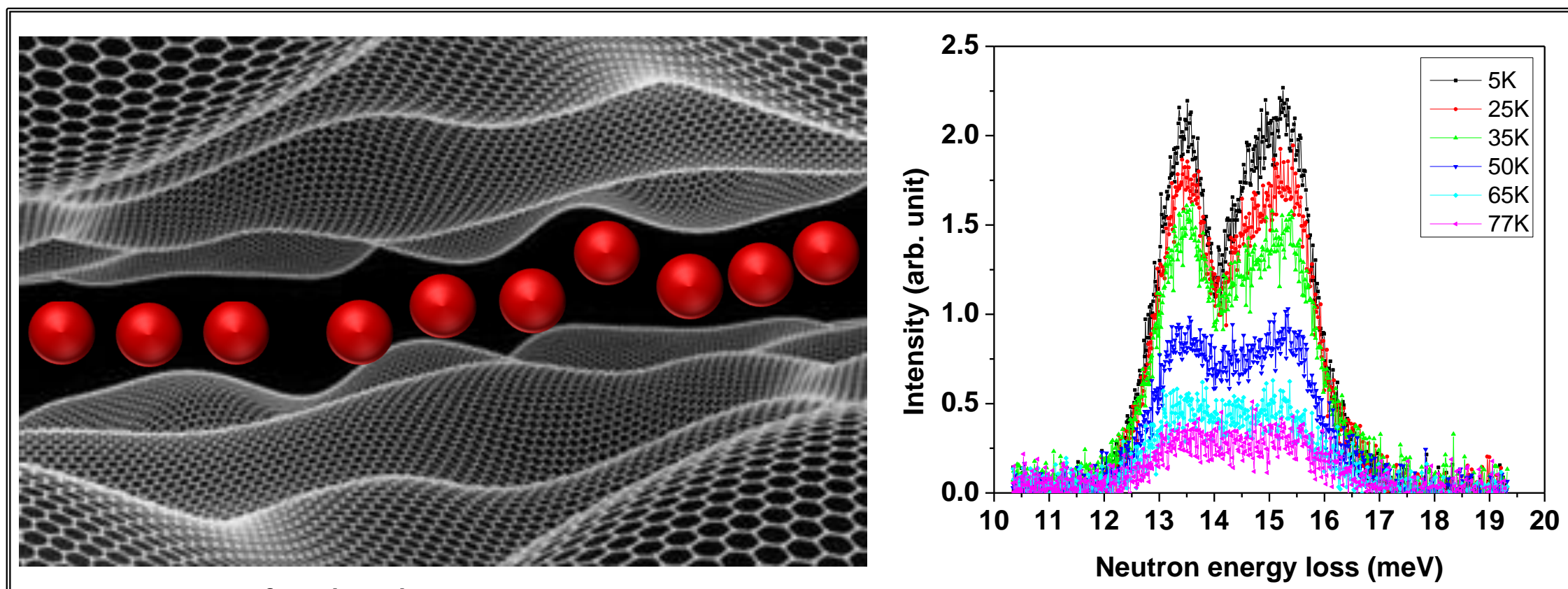

Confined Hydrogen in micropores 